\title{
The relationship between 68Ga-PSMA uptake and Gleason Score and PSA levels in patients with prostate cancer
}

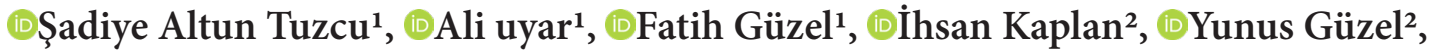 \\ (D)Mansur Dağgülli ${ }^{3}$, ĐBekir Taşdemir ${ }^{1}$ \\ ${ }^{1}$ Dicle University, Faculty of Medicine, Department of Nuclear Medicine, Diyarbakır, Turkey \\ ${ }^{2}$ Diyarbakır Gazi Yaşargil Research and Education Hospital, Department of Nuclear Medicine, Diyarbakır, Turkey \\ ${ }^{3}$ Dicle University, Faculty of Medicine, Department of Urology, Diyarbakır, Turkey
}

Cite this article as: Altun Tuzcu Ş, Uyar A, Güzel F, et al. The relationship between 68Ga-PSMA uptake and Gleason Score and PSA levels in patients with prostate cancer. Anatolian Curr Med J 2021; 3(4); 327-332.

\begin{abstract}
Aim: PSMA expression has been observed in increased levels in patients with high Gleason scores. Current information on Ga-68 PSMA PET/CT shows that primary staging with PET/CT is important in patients with high-risk PC. Ga-68 PSMA PET/ CT may also have a place in patients with intermediate risk PC, but only a few data are available at present. In this study, we aimed to elucidate the relationship between PSMA expression value in the prostate gland, total PSA levels and GS in patients diagnosed with prostate cancer.

Material and Method: A total of 98 patients who were pathologically diagnosed with prostate cancer that did not receive any treatment and underwent Ga-68 PSMA PET/CT imaging for staging were included in the study. Findings detected in Ga-68 PSMA PET/CT imaging were categorized as prostate, lymph node, bone, and visceral organ. The focal increased PSMA expression values observed in the prostate gland were recorded as SUVmax. The patients were divided into two separate groups according to their GS score (GS>7 and GS $\leq 7$ ). Correlations between prostate PSMA SUVmax, GS score and total PSA scores were investigated.

Results: PSMA SUVmax levels of the group with a Gleason score of $>7$ were found to be significantly higher than the group with a Gleason score of $\leq 7(\mathrm{p}=0.03)$. The rates of lymph node metastasis, bone metastasis and visceral organ metastases were found to be significantly higher in the group with a Gleason score $>7$ compared to the group with a Gleason score $\leq 7$. A positive correlation was observed between PSMA SUVmax and Total PSA $(r=0.260, p=0.01)$. A positive correlation was observed between PSMA SUVmax and Gleason score $(\mathrm{r}=0.260, \mathrm{p}=0.01)$. A positive correlation was observed between total PSA and Gleason score $(\mathrm{r}=0.320, \mathrm{p}=0.001)$.

Conclusion: In conclusion, PSMA SUVmax and Total PSA levels were higher in the group with high Gleason score. There is a positive correlation between PSMA SUVmax and total PSA. Clinicians should be careful in this regard, as the possibility of metastasis will be high in groups with high Gleason scores.
\end{abstract}

Keywords: Prostate specific membrane antigen, prostate cancer, Ga-68 PSMA PET/CT

\section{INTRODUCTION}

Prostate cancer (PC) is the second most common type of cancer seen in men all over the world and ranks fourth among all cancers (1). While the 5-year survival rate is approximately $100 \%$ in patients with localized PC, this rate drops to $31 \%$ in patients with distant metastases (2). Despite all the advances in early diagnosis and treatment, including prostate-specific antigen (PSA) screening as well as imaging methods and genetic analysis, prostate cancer is the second leading cause of cancer-related death in men after lung cancer. A significant proportion of prostate cancer patients are metastatic at the time of diagnosis.
PSMA has been an ideal molecular target for both the diagnosis and treatment (theranostic) of prostate cancer in nuclear medicine. The intense expression of PSMA in prostate cancer cells is up to 1000 times compared to normal prostate cells, which is not released freely into the circulation. This constitutes an important molecular target in the diagnosis and treatment of metastatic castration resistant prostate cancers (mCRPC) (3).

In general, increased PSMA expression is observed in poorly differentiated, metastatic and hormone- 
refractory PC. PSMA expression has been observed in increased levels in patients with high Gleason scores. Involvement is directly proportional to aggressiveness, metastasis and hormone therapy resistance and is an important indicator for prognosis (4). Current information on Ga-68 PSMA PET/CT shows that primary staging with $\mathrm{PET} / \mathrm{CT}$ is important in patients with high-risk PC.

It was previously stated that Ga-68 PSMA PET/CT is a more appropriate technique for detecting the focus or foci of recurrence, especially at low PSA levels (0.2-2.0 ng/ $\mathrm{mL}$ ) after primary treatment, compared to conventional imaging techniques. The sensitivity of Ga-68 PSMA PET/ CT increases in relation to GS and PSA kinetics (PSA doubling time) (5).

The most important advantage of Ga-68 PSMA is that it has been shown to be superior to F-18 choline and other currently FDA-approved agents (C-11 choline, F-18 Flucyclovin) in PET imaging at low PSA values in detecting PC recurrence (mean sensitivity 76\%-86, specificity 86-100\%) (6). Although there is no FDA approval for the recommendation of Ga-68 PSMA PET/CT imaging for PC, in the current version of the National Comprehensive Cancer Network (NCCN), it can be performed in clinical trials and controlled studies (6). According to the EAU guideline, it is recommended after radical prostatectomy (RP), and in biochemical recurrence $(\mathrm{BR})$ in radiotherapy $(\mathrm{RT})$.

Finally, in terms of "optimal imaging in advanced PC" in ASCO guideline, there are recommendations under the definition of new generation imaging (NGI) as PSMA PET imaging in addition to conventional imaging under the conditions specified in advanced PC (7).

\section{Study Hypothesis}

In this study, we aimed to elucidate the relationship between PSMA expression value in the prostate gland, total PSA levels and GS in patients diagnosed with prostate cancer.

\section{MATERIAL AND METHOD}

The study was carried out with the permission of Dicle University Faculty of Medicine Non-interventional Clinical Research Ethics Committee (Date: 2021, Decision No: 359). All procedures were carried out in accordance with the ethical rules and the principles of the Declaration of Helsinki.

A total of 98 patients who were pathologically diagnosed with prostate cancer and applied to Dicle University Faculty of Medicine, Department of Nuclear Medicine between April 2019 and May 2021 has been retrospectively analyzed. Informed consent has been obtained from all participants.
Individuals that did not receive any treatment and underwent Ga-68 PSMA PET/CT imaging for staging were included in the study. Findings detected in Ga-68 PSMA PET/CT imaging were categorized as prostate, lymph node, bone, and visceral organ. The focal increased PSMA expression values observed in the prostate gland (with or without corresponding lesions on CT) were recorded as SUVmax. The maximum standardized uptake value (maximum standardized uptake value-SUVmax) values of all lesions that were considered positive were measured and recorded. In addition, lymph nodes, bones and visceral organs were evaluated whether there was metastasis according to PSMA involvement. Imaging with any pathological finding was considered positive, and imaging without any pathological finding was considered negative. Except for the areas of physiological involvement, the involvement above the ground activity was considered significant.

Areas with involvement were anatomically localized with nondiagnostic CT images. The pathology reports of the patients were evaluated, and the Gleason score (GS) values were recorded. Prostate Specific Antigen (PSA) data measured in the last 1 month according to the imaging date of the patients were obtained.

In addition, the patients were divided into two separate groups according to their GS score (GS>7 and GS $\leq 7$ ). We investigated whether there were differences in total PSA, Prostate PSMA SUVmax values between these two groups. In addition, correlations between prostate PSMA SUVmax, GS score and total PSA scores were investigated.

Ga-68 PSMA PET/CT images of the patients were evaluated by two Nuclear Medicine specialists.

\section{Statistical Analysis}

IBM SPSS 21.0 for Windows statistical package program was used for the statistical evaluation of the data. Measurable variables were presented as mean \pm standard deviation, and categorical variables as numbers and percentages $(\%)$. The chi-square $\left(\chi^{2}\right)$ test was used for comparing categorical variables. Independent- $t$ test was used for comparing measurable variables. In addition, Spearman correlation test was utilized for the correlation between Gleason score and PSMA SUVmax and total PSA. Pearson correlation test was performed to examine the correlation between PSMA SUVmax and total PSA. A P value $\leq 0.05$ indicated statistically significant difference.

\section{RESULTS}

The mean ages were not different between the group with a Gleason score of $\leq 7$ and the group with a Gleason score of $>7$. 
Total PSA level was found to be significantly higher in the group with Gleason score $>7$ than in the group with Gleason level $\leq 7(\mathrm{p}=0.001)$.

PSMA SUVmax levels of the group with a Gleason score of $>7$ were found to be significantly higher than the group with a Gleason score of $\leq 7(\mathrm{p}=0.03)$ (Table 1).

\begin{tabular}{|c|c|c|c|}
\hline & $\begin{array}{c}\text { Gleason score } \leq 7 \\
(\mathbf{n}=50)\end{array}$ & $\begin{array}{c}\text { Gleason score }>7 \\
(n=48)\end{array}$ & $P$ value \\
\hline Age (years) & $65.40 \pm 7.1$ & $65.3 \pm 7.6$ & $>0.05$ \\
\hline Total PSA & $28.5 \pm 33.6$ & $52.6 \pm 39.7$ & 0.001 \\
\hline PSMA SUVmax & $11.8 \pm 7.6$ & $15.2 \pm 7.89$ & 0.03 \\
\hline
\end{tabular}

The rates of lymph node metastasis, bone metastasis and visceral organ metastases were found to be significantly higher in the group with a Gleason score $>7$ compared to the group with a Gleason score $\leq 7$ (Table 2).

\begin{tabular}{|c|c|c|c|}
\hline & $\begin{array}{c}\text { Gleason score } \\
\leq 7(\mathbf{n}=50)\end{array}$ & $\begin{array}{l}\text { Gleason score } \\
\quad>7(n=48)\end{array}$ & $P$ value \\
\hline $\begin{array}{l}\text { Lymph node } \\
\text { metastasis (yes/no) }\end{array}$ & $\begin{array}{l}16(32 \%) / \\
34(68 \%)\end{array}$ & $\begin{array}{l}32(66.7 \%) / \\
16(33.3 \%)\end{array}$ & 0.001 \\
\hline $\begin{array}{l}\text { Bone metastasis } \\
\text { (yes/no) }\end{array}$ & $\begin{array}{l}12(24 \%) / \\
38(76 \%)\end{array}$ & $\begin{array}{l}27(56.3 \%) / \\
21(43.8 \%)\end{array}$ & 0.002 \\
\hline $\begin{array}{l}\text { Visceral organ } \\
\text { metastasis (yes/no) }\end{array}$ & $\begin{array}{c}1(2 \%) / \\
49(98 \%)\end{array}$ & $\begin{array}{l}8(16.7 \%) / \\
40(93.7 \%)\end{array}$ & 0.01 \\
\hline
\end{tabular}

A positive correlation was observed between PSMA SUVmax and Total PSA ( $\mathrm{r}=0.260, \mathrm{p}=0.01)$ (Figure 1).

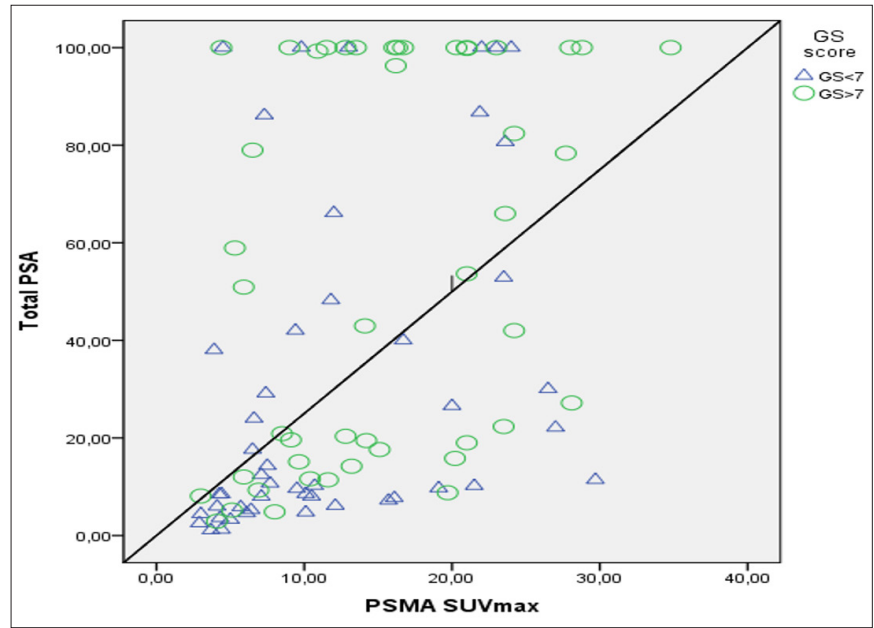

Figure 1. Scatter graph showing the correlation between Total PSA and PSMA SUV max

A positive correlation was observed between PSMA SUVmax and Gleason score $(r=0.260, p=0.01)$. A positive correlation was observed between total PSA and Gleason score $(\mathrm{r}=0.320, \mathrm{p}=0.001)$ (Table 3)(Figure 2).
Tablo 3. Correlation between Gleason score PSMA SUVmax and Total PSA

\begin{tabular}{|lcc|}
\hline & Gleason score & Total PSA \\
\hline PSMA SUVmax & $\mathrm{R}=0.260 . \mathrm{p}=0.01$ & $\mathrm{R}=0.524 . \mathrm{p}=0.001$ \\
Total PSA & $\mathrm{R}=0.320 \mathrm{p}=0.001$ & \\
\hline
\end{tabular}

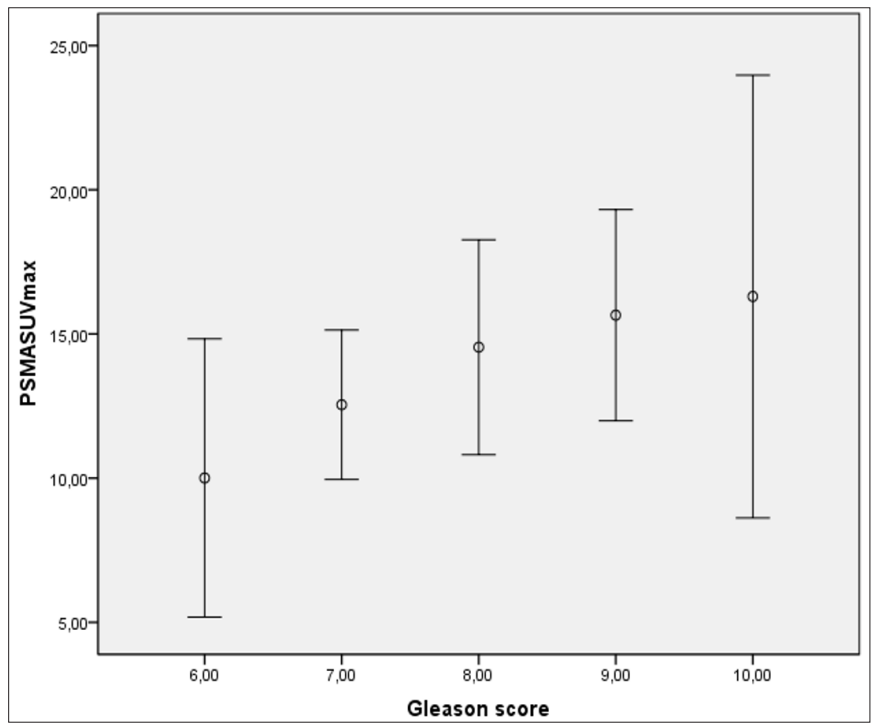

Figure 2. Correlation between PSMA SUVmax and Gleason score

\section{DISCUSSION}

Ga-68 PSMA PET/CT is being used more and more frequently in the staging of prostate cancer, determining the treatment plan, and detecting the recurrent lesion and its localization even at low PSA levels in patients with biochemical recurrence. Studies evaluating the relationship between the findings in Ga-68 PSMA PET/ $\mathrm{CT}$, pathology and laboratory parameters are increasing day by day. These studies also derive a significant relationship was between PSMA involvement and GS $(8,9)$.

The rates of lymph node metastasis, bone metastasis and visceral organ metastases were found to be significantly higher in the group with a Gleason score $>7$ compared to the group with a Gleason score $\leq 7$. In a study, it was reported that distal metastases such as lymph nodes, bones and liver were observed more frequently in patients with a Gleason score above 7. These findings are consistent with the results of our study (10). In a retrospective series, the sensitivity and specificity of Ga-68 PSMA was reported as $98-100 \%$ and $88-100 \%$, respectively, for the detection of skeletal metastases at the initial staging of PC, while it was reported as $86-89 \%$ and $60-96 \%$ in bone scintigraphy (11). Similarly, Thomas et al (12). showed that Ga-68 PSMA was superior to bone scintigraphy in detecting skeletal metastases in PC, and Ga-68 PSMA detected approximately twice as many lesions. In a recent study, Ga-68 PSMA PET/CT had significantly higher sensitivity and accuracy than bone scintigraphy $(96.2 \%$ vs. $73.1 \%$ and $99.1 \%$ vs. $84 \%$ ). 1 ) has been reported to 
show. Therefore, current guidelines recommend the use of PSMA PET/CT to enable early detection of recurrences and salvage treatment options $(4,13)$.

In this study we have found that the total PSA level was significantly higher in the group with Gleason score $>7$ than in the group with Gleason level $\leq 7(\mathrm{p}=0.001)$. PSMA SUVmax levels of the group with a Gleason score of $>7$ were found to be significantly higher than the group with a Gleason score of $\leq 7(\mathrm{p}=0.03)$. In previous literature, Ga-68 PSMA PET/CT imaging is recommended for patients diagnosed with prostate cancer and those in the medium-high risk group $(14,15)$. In this study, according to the D'Amico risk classification, 33 staging patients were in the high risk group and 7 were in the intermediate risk group (TRUS-Bx was performed 3 times in one patient and adenocarcinoma was diagnosed in the last biopsy, Gleason score was not given in the report). Uprimny et al. (16), in their study with 90 patients diagnosed with prostate cancer, found that SUVmax values measured from the prostates of patients with GS 7 and below (GG3 and below) and patients with a GS above 7 (GG3 and above) differed significantly. In the study of Uprimny et al. (16), a significant difference was found between patients with a PSA value of $10 \mathrm{ng} / \mathrm{ml}$ and below and patients with a PSA value above $10 \mathrm{ng} / \mathrm{ml}$ in terms of prostate SUVmax values.

We have also found relation of PSA and other variables. In this study a positive correlation was observed between PSMA SUVmax and Total PSA $(\mathrm{r}=0.260, \mathrm{p}=0.01)$. A positive correlation was observed between PSMA SUVmax and Gleason score $(\mathrm{r}=0.260, \mathrm{p}=0.01)$. A positive correlation was observed between total PSA and Gleason score $(\mathrm{r}=0.320, \mathrm{p}=0.001)$. In a study by Klingenberg et al. (17) a significant positive correlation was found between prostate PSMA SUVmax values and International Society of Urological Pathology (ISUP) grade. In the same study, a positive correlation between PSA levels and prostate PSMA SUVmax was shown, which is consistent with the findings of our study. PSMA SUVmax and Total PSA levels were higher in the group with high Gleason score. There is a positive correlation between PSMA SUV max and total PSA. Eiber et al. (18) retrospectively evaluated 248 patients with prostate cancer diagnosed with biochemical recurrence after radical prostatectomy. In this study, pathological findings were detected in Ga68 PSMA PET/CT imaging in $89.5 \%$ of the patients. They found that PSA values differed significantly between patients with negative imaging and patients with positive imaging. In this study, a significant difference was found between PSA values. Eiber et al. (18) also found that the Ga-68 PSMA PET/CT positivity rates of patients with GS 7 and below and patients with 8 and above differed significantly (18).
In a study by Chun et al. (19), the results of transrectal US-guided prostate biopsy (TRUS-Bx) and radical prostatectomy results of 2982 prostate cancer patients were compared and GS upgrade has been detected in $29 \%$ of the patients and downgrade in $14 \%$ of the patients. It was thought that this might be one of the reasons why there was no correlation between SUVmax values and GS/GG values. In fact, this finding suggests that the prognosis and treatment of the patient may change due to the lower reporting of GS/GG in patients diagnosed with TRUS-Bx, and SUVmax value can be used as a more valuable prognostic factor than GS/GG (19). Ceci et al. (20) retrospectively analyzed 70 patients with prostate cancer who had undergone radical prostatectomy or received radical RT for primary malignancy and had biochemical recurrence or persistent PSA elevation and therefore had Ga-68 PSMA PET/CT imaging. They accepted the imaging as positive Ga-68 PSMA PET/ CT in which they detected any pathological finding. Accordingly, 74\% of the patients are positive for Ga-68 PSMA PET/CT (20).

Obek et al. (20) found the sensitivity, specificity, and accuracy in the subgroup with $\geq 15$ lymph nodes removed with histopathological correlation $(n=37)$ were $67 \%, \%$, respectively. 88 and $81 \%$, morphological imaging (MR, CT) found $20 \%, 99 \%$ and $72 \%$ in primary lymph node staging of Ga-68 PSMA PET/CT in 51 newly diagnosed high-risk PC patients. The authors stated that Ga-68 PSMA PET/CT is superior to morphological imaging in detecting lymph node metastasis, while surgical dissection remains the gold standard for lymph node staging. Therefore, Ga-68 PSMA PET/CT can replace conventional abdominal-pelvic CT in staging lymph node metastasis in PC (21).

In the ASCO guideline, although the role of new generation imaging including Ga-68 PSMA PET/CT in non-metastatic CRPC is not clear due to limited data, it is superior to conventional techniques in demonstrating metastatic, non-metastatic disease and will contribute to patient management with local treatment strategies $(22,23)$. In addition, the role of Ga-68 PSMA PET/ $\mathrm{CT}$ is not yet known when there is PSA progression in metastatic CRPC and if conventional imaging is negative, and although it is not routinely recommended when there is radiological progression, it is stated that it may have a role in evaluating the extent of progression and monitoring the findings $(7,22)$. In prospective and retrospective studies, it has been reported that Ga-68 PSMA PET/CT, with its high positive predictive value and recurrence detection rate at low PSA levels, causes up to $50 \%$ change in patient management in general $(24,25)$. 


\section{Limitations of the Study}

The main limitation of the study could be attributed to its retrospective nature. The other limitation was elaborated as single-center patient enrollment but this might be rationalized by the retrospective data collection.

The strength of this study lies beneath the fact that staging patients who have not received any treatment were included in the analysis. The other upside could be emphasized as it is an emerging era.

\section{CONCLUSION}

In conclusion, PSMA SUVmax and Total PSA levels were higher in the group with high Gleason score. There is a positive correlation between PSMA SUV $\max$ and total PSA. Clinicians should be careful in this regard, as the possibility of metastasis will be high in groups with high Gleason scores. According to the results of this study one can say that Ga-68 PSMA PET/ CT imaging for staging suggests that it should be put into routine use in medium-high risk patients during staging purposes. Regarding the association between Ga-68 PSMA PET/CT findings and SUVmax values, histopathological findings and laboratory findings, SUVmax values are also can be used as a prognostic factor.

\section{ETHICAL DECLARATIONS}

Ethics Committee Approval: The study was carried out with the permission of Dicle University Faculty of Medicine Non-interventional Clinical Research Ethics Committee (Date: 2021, Decision No: 359).

Informed Consent: Because the study was designed retrospectively, no written informed consent form was obtained from patients

Referee Evaluation Process: Externally peer-reviewed. Conflict of Interest Statement: The authors have no conflicts of interest to declare.

Financial Disclosure: The authors declared that this study has received no financial support.

Author Contributions: All of the authors declare that they have all participated in the design, execution, and analysis of the paper, and that they have approved the final version.

\section{REFERENCES}

1. Ferlay J, Soerjomataram I, Dikshit R, et al. Cancer incidence and mortality worldwide: sources, methods and major patterns in GLOBOCAN 2012. Int J Cancer 2015; 136: 359-86.

2. 2.Siegel RL, Sahar L, Portier KM, et al. Cancer death rates in US congressional districts. CA Cancer J Clin 2015; 65: 339-44.
3. Thomas L, Balmus C, Ahmadzadehfar H, Essler M, Strunk H, Bundschuh RA. Assessment of bone metastases in patients with prostate cancer-a comparison between (99m)Tc-bonescintigraphy and [(68)Ga]Ga-PSMA PET/CT. Pharmaceuticals (Basel) 2017; 31: 10 .

4. Lengana T, Lawal IO, Boshomane TG, et al. (68)Ga-PSMA PET/ CT replacing bone scan in the initial staging of skeletal metastasis in prostate cancer: a fait accompli? Clin Genitourin Cancer 2018; 16: 392-401.

5. Verburg FA, Pfister D, Heidenreich A, et al. Extent of disease in recurrent prostate cancer determined by [(68)Ga]PSMA-HBEDCC PET/CT in relationto PSA levels, PSA doubling time and Gleason score. Eur J Nucl Med Mol Imaging 2016; 43: 397- 403.

6. National Comprehensive Cancer Network. NCCN Clinical Guidelines in Oncology. Prostate Cancer. Version 2.2020. 2020; https://www.nccn.org/professionals/physician_gls/pdf/prostate. pdf

7. Fendler WP, Eiber M, Beheshti M, et al. 68Ga-PSMA PET/ CT: Joint EANM and SNMMI procedure guideline for prostate cancer imaging: version 1.0. Eur J Nucl Med Mol Imaging 2017; 44: 1014-24.

8. Kirby M, Hirst C, Crawford ED. Characterising the castrationresistant prostate cancer population: a systematic review. Int J Clin Pract 2011; 65: 1180-92.

9. Siegel RL, Sahar L, Portier KM, et al. Cancer death rates in US congressional districts. CA Cancer J Clin 2015; 65: 339-44.

10. Topuz ÖV, Aksu A, Erinç SR, Tamam MÖ.Correlations of 68 GaPSMA PET/CT in the initial staging of prostate cancer patients. Hell J Nucl Med 2021; 24: 60-65.

11. Pyka T, Okamoto S, Dahlbender M, et al. Comparison of bone scintigraphy and (68)Ga-PSMA PET for skeletal staging in prostate cancer. Eur J Nucl Med Mol Imaging 2016; 43: 2114-21.

12. Sridhar SS, Freedland SJ, Gleave ME, et al. Castration-resistant prostate cancer: from new pathophysiology to new treatment. Eur Urol 2014; 65: 289-99.

13. Cornford P, Bellmunt J, Bolla M, et al. EAU-ESTRO-SIOG guidelines on prostate cancer. Part II: treatment of relapsing, metastatic, and castration-resistant prostate cancer. Eur Urol 2017; 10

14. Bailey J, Piert M. Performance of 68Ga-PSMA PET/CT for prostate cancer management at initial staging and time of biochemical recurrence. Curr Urol Rep 2017; 18: 84.

15. Lenzo NP, Meyrick D, Turner JH. Review of Gallium-68 PSMA PET/CT imaging in the management of prostate cancer. Diagnostics (Basel, Switzerland) 2018; 8: 16.

16. Uprimny C, Kroiss AS, Decristoforo C, et al. 68Ga-PSMA-11 PET/CT in primary staging of prostate cancer: PSA and Gleason score predict the intensity of tracer accumulation in the primary tumour. Eur J Nucl Med Mol Imaging 2017; 44: 941-9.

17. Klingenberg S, Jochumsen MR, Ulhøi BP, et al. (68)Ga-PSMA PET/CT for primary lymph node and distant metastasis NM staging of high-risk prostate cancer. J Nucl Med 2021; 62: 214-20.

18. Eiber M, Maurer T, Souvatzoglou M, et al. Evaluation of hybrid 68Ga-PSMA ligand PET/CT in 248 patients with biochemical recurrence after radical prostatectomy. J Nucl Med 2015; 56: 668-74.

19. Chun FK-H, Steuber T, Erbersdobler A, et al. Development and internal validation of a nomogram predicting the probability of prostate cancer Gleason sum upgrading between biopsy and radical prostatectomy pathology. Eur Urol. 2006; 49: 820-6.

20. Ceci F, Uprimny C, Nilica B, et al. (68)Ga-PSMA PET/CT for restaging recurrent prostate cancer: which factors are associated with PET/CT detection rate? Eur J Nucl Med Mol Imag 2015; 42: 1284-94. 
21. Öbek C, Doğanca T, Demirci E, et al. Members of Urooncology Association, Turkey. the accuracy of 68Ga-PSMA PET/CT in primary lymph node staging in high-risk prostate cancer. Eur J Nucl Med Mol Imaging 2017; 44: 1806-12.

22. Mottet N, Bellmunt J, Briers E. et al. Members of the EAUESTRO-ESUR-SIOG Prostate Cancer Guidelines Panel. EAUESTRO-ESUR-SIOG Guidelines on Prostate Cancer. Edn. presented at the EAU Annual Congress Amsterdam 2020. 97894-92671-07-3. Arnhem: EAU Guidelines Office; 2020.

23. Calais J, Fendler WP, Eiber M, et al. Impact of 68Ga-PSMA$11 \mathrm{PET} / \mathrm{CT}$ on the management of prostate cancer patients with biochemical recurrence. J Nucl Med 2018; 59: 434-41.

24. Sonni I, Eiber M, Fendler WP, et al. Impact of 68Ga-PSMA-11 $\mathrm{PET} / \mathrm{CT}$ on staging and management of prostate cancer patients in various clinical settings: a prospective single center study. J Nucl Med 2020; 61: 1153-60.

25. Uprimny C, Kroiss AS, Decristoforol C, et al. 68Ga-PSMA-11 PET/CT in primary staging of prostate cancer: PSA and Gleason score predict the intensity of tracer accumulation in the primary tumour, Eur J Nucl Med Mol Imaging 2017; 44: 941-9. 